\title{
Food cravings, binge eating and emotional eating behaviours in overweight and obese women with polycystic ovary syndrome
}

\author{
A. Morosi and Y. Jeanes \\ Health Sciences Research Centre, University of Roehampton, London
}

Polycystic ovary syndrome (PCOS) is the most common endocrine disorder affecting reproductive-age women ${ }^{(1)}$, and its symptoms are exacerbated by insulin resistance and obesity ${ }^{(2)}$. One of the major challenges for women with PCOS is weight management, which could be influenced by the anecdotally reported food cravings and emotional eating behaviours ${ }^{(3)}$. The study aimed to explore associations between food cravings, binge eating and emotional eating behaviours in overweight and obese women with PCOS.

Participants completed a web-based survey, which included the Food Craving Questionnaire-Trait (FCQ-T) ${ }^{(4)}$, the Three Factor Eating Questionnaire Revised 18-item, Version $2^{(5)}$, and the Bulimic Investigatory Test (BITE) ${ }^{(6)}$. Ethical approval was granted through the procedures of the University of Roehampton.

Mean (SD) binge eating (BITE) score from 338 overweight and obese women with PCOS was $23 \cdot 9(8 \cdot 7)$, food craving (FCQ-T) score was $130 \cdot 4(29 \cdot 0 ; n=343)$ and emotional eating score was $2 \cdot 7(0 \cdot 8 ; n=334)$. Binge eating scores were strongly associated with FCQ-T scores $(r=0.65, \mathrm{P}<0.001)$ and emotional eating scores $(\mathrm{r}=0.56, \mathrm{P}<0.001)$. Overweight and obese women with PCOS who had previously been successful in reducing their body weight reported lower binge eating scores $(19 \cdot 8(7 \cdot 9)$ versus $26 \cdot 7(8 \cdot 2)$, $\mathrm{P}<0.001)$ and lower scores on the lack of control over eating subscale from the FCQ-T $(18.7(5 \cdot 8)$ versus $21 \cdot 3(5.4), \mathrm{P}<0.001)$.

This large cohort of overweight and obese women with PCOS present with greater food cravings than non PCOS population ${ }^{(7)}$ and more binge eating ${ }^{(8)}$. Disordered eating behaviours may be a contributor to the difficulties with weight management reported amongst women with PCOS or the results of persistent dieting behaviours; further research is needed to elucidate the direction of this effect. It is important that clinicians are aware of food cravings and binge eating behaviours in women with PCOS to help ensure appropriate weight loss strategies are communicated to women with PCOS. Success of weight-loss interventions could be maximized by combining lifestyle advice with psychological therapy.

1. March WA, Moore VM, Willson KJ et al. (2010) The prevalence of polycystic ovary syndrome in a community sample assessed under contrasting diagnostic criteria. Hum Reprod 25, 544-551.

2. Lim SS, Norman RJ, Davies MJ et al. (2013) The effect of obesity on polycystic ovary syndrome: a systematic review and meta-analysis. Obes Rev 14, $95-109$.

3. Moroshko I, Brennan L, O’Brien P (2011) Predictors of dropout in weight loss interventions: a systematic review of the literature. Obes Rev 12, 912-934.

4. Cepeda-Benito A, Gleaves DH, Williams TL et al. (2000) The development and validation of the state and trait food-cravings questionnaires. Behav Ther 31, 151-173.

5. Cappelleri JC, Bushmakin AG, Gerber RA et al. (2009) Psychometric analysis of the Three-Factor Eating Questionnaire-R21: results from a large diverse sample of obese and non-obese participants. Int $J$ Obes 33, 611-620.

6. Henderson M \& Freeman CP (1987) A self-rating scale for bulimia. The 'BITE'. Br J Psychiatry 150, 18-24.

7. Vander Wal JS, Johnston KA, Dhurandhar NV (2007) Psychometric properties of the State and Trait Food Cravings Questionnaires among overweight and obese persons. Eat Behav 8, 211-223.

8. Moreno S, Warren CS, Rodriguez S et al. (2009) Food cravings discriminate between anorexia and bulimia nervosa. Implications for "success" versus "failure" in dietary restriction. Appetite 52, 588-594. 